\title{
Thermo-physical properties of synthetic mucus for the study of airway clearance
}

\author{
O. Lafforgue, ${ }^{1}$ N. Bouguerra, ${ }^{2}$ S. Poncet $\mathbb{D}^{1},{ }^{1,2}$ I. Seyssiecq, ${ }^{1}$ J. Favier $\mathbb{C}^{1},{ }^{1}$ S. Elkoun ${ }^{2}$ \\ ${ }^{1}$ Aix-Marseille Université, CNRS, Ecole Centrale de Marseille, Laboratoire M2P2 UMR 7340, 38 rue F. Joliot-Curie, Technopôle \\ de Château-Gombert, Marseille, 13451, France \\ ${ }^{2}$ Université de Sherbrooke, Faculté de génie, Département de génie mécanique, 2500 Boulevard de I'Université, Sherbrooke, \\ OC, J1K 2R1, Canada
}

\begin{abstract}
In this article, dynamic viscosity, surface tension, density, heat capacity and thermal conductivity, of a bronchial mucus simulant proposed by Zahm et al., Eur Respir J 1991; 4: 311-315 were experiementally determined. This simulant is mainly composed of a galactomannan gum and a scleroglucan. It was shown that thermophysical properties of synthetic mucus are dependant of scleroglucan concentrations. More importantly and for some scleroglucan concentrations, the syntetic mucus, exhibits, somehow, comparable thermophysical properties to real bronchial mucus. An insight on the microstructure of this simulant is proposed
\end{abstract}

and the different properties enounced previously have been measured for various scleroglucan concentrations and over a certain range of operating temperatures. This synthetic mucus is found to mimic well the rheological behavior and the surface tension of real mucus for different pathologies. Density and thermal properties have been measured for the first time. (C) 2017 Wiley Periodicals, Inc. J Biomed Mater Res Part A: 105A: 3025-3033, 2017.

Key Words: synthetic mucus, cystic fibrosis, rheology, thermo-physical properties

\section{INTRODUCTION}

Mucus is a complex biological material whose role in human health is to lubricate and protect organs such as lungs, vagina, eyes or gastrointestinal tract among others. It acts as a physical barrier against pathogens and noxious particles $^{2}$ while preventing tissue dehydration and allowing useful molecules (for example, ions or proteins) to be transferred through.

The present article focuses on the thermophysical properties of a bronchial mucus simulant. Many respiratory diseases such as cystic fibrosis (CF) are related to abnormal compositions of lung mucus related to a default of transfer for certain ions through the epithelial surface. This results in a hyper viscosity of the mucus, impairing the process of mucociliary clearance, and exacerbating inflammations and chronic infections. As reported by Flume et al., ${ }^{3}$ there is to date, no clear consensus about the most efficient therapy to support the airway clearance and treat adequately patients suffering from $\mathrm{CF}$ or chronic obstructive pulmonary disease (COPD). Presently, most physicians used a combination of drugs ${ }^{4}$ respiratory physiotherapy (with a therapist and/or an airway clearance device) and regular physical activities.
In addition, some devices were designed to increase mucus secretion volume to help the airway clearance and increase the patient autonomy. Either based on vibrations, percussions or positive expiratory pressures, the functioning of these devices is based on the shear-thinning and thixotropic properties of bronchial mucus. A given shear stress is applied on the chest or during the exhalation phase of the respiratory cycle, increasing mucus fluidity, thus helping patients to expectorate it more easily. One could cite as examples the Frequencer ${ }^{\circledR},{ }^{5}$ which transmits acoustic and mechanical sinusoidal vibrations at different locations inside the chest, or more recently the Simeox ${ }^{\circledR}$, which imposes an oscillatory air depression during the exhalation phase. ${ }^{6}$

Mucus exhibits a complex structure with different characteristic scales. ${ }^{7}$ It is made of $90-95 \%$ water, $2-5 \%$ mucins, $1-2 \%$ lipids, $1 \%$ salts, and $0.02 \%$ of DNA and other molecules such as cells debris. ${ }^{8}$ It forms a physical gel structured by a three dimensional mucin matrix. Mucins are high molecular weight glycoproteins (length 0.5-10 $\mu \mathrm{m}$ ) insuring a structural protection function. Within the airways, they can be found as two types ${ }^{9}$ : monomeric and oligomeric mucins, the latter are able to form a gel and are 
mainly responsible for the complex rheological properties of the mucus gel. In human native mucus, the mucins of the gel-forming type (mainly MUC2, MUC5AC, MUC5B and MUC6) build with other proteins, a three dimensional gel network. These mucins consist in a peptide chain with 0 glycosylated regions (branched sugar chain, endowing the gel-forming ability) and cysteine-rich naked regions (non glycosylated or N-glycosylated). The mucins are oligomerized by strong disulfide inter-molecular bonds; there are also disulfide intra-molecular bonds which stabilize the naked domains. These latter branch free domains are hydrophobic and entail the cross-link of two naked mucin regions trying to avoid water. In addition to these weak hydrophobic bonds and the strong disulfide bonds, several interactions play a role in the cross-linked network: entanglements due to the macromolecules large sizes, hydrogen bonds between sugar branches which are weak but numerous and Van der Waals forces between oligosaccharide moieties. ${ }^{10}$ Boat et al. ${ }^{11}$ have summarized the biochemistry of mucus, while Verdugo ${ }^{12}$ provided a deep insight into the dynamics of the different mucus components.

At a macroscopic scale, mucus is also a non-Newtonian fluid, exhibiting properties of viscoelasticity, shear-thinning and thixotropy, while, at a microscale, it behaves as a low viscosity fluid. The reader can refer to Rubin ${ }^{13}$ or Lai et al. ${ }^{7}$ and the references herein for a state-of-the-art up to 2009 on the micro- and macro-rheology of different types of mucus, including humans and animal ones. One focuses here only on human bronchial mucus from patients suffering from CF or COPD, for which rheology has been studied starting from the $1960 \mathrm{~s}^{14,15}$ and correlated with the mucociliary clearance some years later. ${ }^{16}$ As an example, Puchelle et al. ${ }^{17}$ measured the biochemical and rheological properties of bronchial mucus from 21 chronic bronchitic patients without any medical treatment. The rheological properties of sputum are found to be affected by interactions between its different constituents, namely proteins (like the secretory immunoglobulins, serum albumin, transferrin), the mucins and the nucleic acids. For this pathology, other authors ${ }^{18}$ also assessed the important role of spinability, together with the ones of viscosity and elasticity, on the mucociliary transport rate. The thixotropic property of bronchial mucus was investigated some years later by the same authors using bronchial mucus and simulants and was interpreted as a modification in the three-dimensional structure at a microscale. ${ }^{19}$ Puchelle et al. ${ }^{20}$ correlated the mucus viscosity and the mucociliary transport. Beyond an optimal value of dynamic viscosity close to 12 Pa.s, the mucociliary frequency and transport rate decrease. The spinability and adhesiveness of mucus are also supposed to modify/regulate the mucociliary clearance. Rubin et al. ${ }^{21}$ characterized the bronchial mucus of healthy patients under two shear rates (1 and $100 \mathrm{rad} / \mathrm{s}$ ) and compared their mechanical impedance and loss tangent with values obtained on other types of mucus sampled from patients suffering from $\mathrm{CF}$, and dogs. Concerning human mucus, they did not notice any difference between men and women nor depending on the age. There was also no significant differences between healthy or CF human mucus nor dogs mucus. Nielsen et al. $^{22}$ performed oscillatory, creep and steady shear rheological measurements over a wide range of characteristic times (from $10^{-3}$ to $10^{6} \mathrm{~s}$ ) using 23 mucus samples from $\mathrm{CF}$ patients. They confirmed that such bronchial mucus exhibits viscoelastic properties with a significant elastic recovery. At low shear rates, a nearly constant steady viscosity is observed for long shearing times. For given shear rates, the measured viscosities are significantly different from the ones previously reported in the literature, highlighting the high variability of sputum characteristics.

The main issues in analyzing bronchial mucus properties can be listed as follows:

1. The difficulty to collect it: on one hand, the collecting method often entails changes in the properties of concern (e.g., saliva contamination, hypersecretion of mucins in response to the collecting tool). ${ }^{7}$ On the other hand, the collectable amount is limited and requires to establish clinical studies.

2. Its huge variability in terms of mucin size, type and concentration. They depend indeed on many factors: patient, pathology (disease conditions, ${ }^{9}$ severity ${ }^{23}$ ) and daily practices (food, smoker/non smoker, ${ }^{24}$ practice of physical activities)...

Hence, many studies are performed on mucus simulants that are engineered in accordance with the properties to be probed (bulk rheology, surface tension, diffusivity...). These mucus models can be produced by cell cultures, which provides a great relevance compared to native mucus but are still limited in quantity, or by the formulation of synthetic gels made of polymeric materials which can be produced in large amounts but hardly gather all mucus properties. ${ }^{25}$ Most of the studies to date on synthetic mucus used either guar gum ${ }^{26}$ or locust bean gum. ${ }^{27-29}$ Madsen et al. ${ }^{30}$ compared four commercial simulants of mucus, Sigma or Orthana mucin types. Banerjee et al. ${ }^{31}$ investigated the influence of three therapeutic surfactants on the viscosity of mucus simulant mainly composed of gum tragacanth. Such polymeric gel was preferred for the presence of fucose, used to simulate fucomucins. The mucus exhibits a shearthinning behavior with a flow index inferior to unity. Shah et al. ${ }^{32}$ developed a simulant based on a polyethylene oxide with addition of resin to test the efficiency of endotracheal suction catheters. Hamed et al. $^{25}$ developed a mucus simulant with a complex composition based on pig gastrin mucins to mimic native tracheal mucus in terms of rheology and surface tension.

The main objective of the present article is to fully characterize the thermophysical properties of a synthetic mucus for different mucin concentrations and operating temperatures. The empirical correlations and different measured properties could then be used for a better understanding of the respiratory diseases impacting the composition of mucus. It could be used to develop better adapted treatments or improve existing numerical models dedicated to 

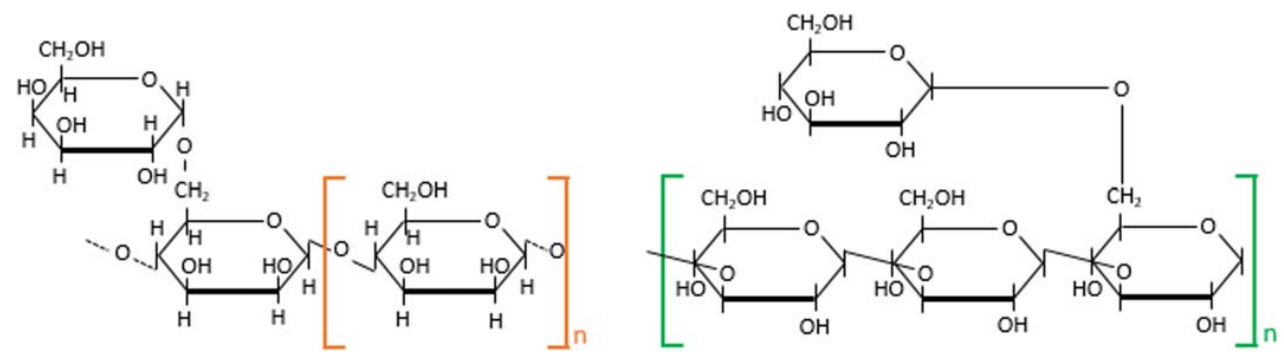

FIGURE 1. Structural chemical formula of (a) Viscogum ${ }^{T M}{ }^{\circledR}$ FA and (b) Actigum ${ }^{T M}{ }^{\circledR}$ CS.

the simulation of airway clearance in human lungs, and go a step forward toward the development of numerical lungs as a predictive tool for medical diagnosis. To the best of the author's knowledge, this experimental study is the first to propose the characterization of the thermal properties and an insight into the structure of a synthetic mucus.

The article is organized as follows: The materials and the preparation of the mucus simulant are described in Section "Measurement Preparation". The techniques used to measure the dynamic viscosity, the density, the surface tension, the heat capacity, the thermal diffusivity and the thermal conductivity are presented in Section "Measurement Techniques". The results are discussed in Section "Results and Discussion" for different mucins concentrations over a given range of temperature before some conclusions in Section "Conclusion".

\section{MATERIALS AND PREPARATION}

\section{Materials}

Viscogum $^{\mathrm{TM}}$ FA (Cargill ${ }^{\mathrm{TM}}$ ), a galactomannan gum derived from locust beans and Actigum ${ }^{\mathrm{TM}} \mathrm{CS} 6$ (Cargill $^{\mathrm{TM}}$ ), a scleroglucan obtained by aerobic fermentation of a Sclerotium fungus were kindly provided by the company Laserson (Etampes, France). Viscogum ${ }^{\mathrm{TM}}$ is composed of galactose and mannose (average of 1 galactose unit for 4 mannose residues). A chain of mannose is branched with galactose sugars irregularly distributed in smooth and substituted zones. Actigum ${ }^{\mathrm{TM}}$ consists in a glucose chain branched every three units by an additional glucose forming a three dimensional (triple helix) structure. Figure 1 shows the molecular structure of these two components. Sodium chloride a.r. $(99.8+\% \mathrm{NaCl})$ and di-sodium tetraborate $10 \mathrm{aq}$ a.r buffer substance $\left(99.5+\% \mathrm{Na}_{2} \mathrm{~B}_{4} \mathrm{O}_{7} \cdot 10 \mathrm{H}_{2} \mathrm{O}\right)$ were purchased from Chem-Lab NV (Zedelgem, Belgium). Distilled water used in all preparations were obtained from a settling tank (GFL 2012).

\section{Preparation of synthetic mucus}

Mucus simulants were prepared within glass bottles filled with $200 \mathrm{mg}$ of distilled water. Each component was then very slowly poured into the solution stirred using a magnetic stirrer (Ikamag® RET) at room temperature (around $21^{\circ} \mathrm{C}$ ). The following protocol taken from Ref. 33 and adapted from Ref. 1 consists in the addition, in the following order, of $0.9 \mathrm{wt} \%$ of $\mathrm{NaCl}, 0.5 \mathrm{wt} \%$ of Viscogum ${ }^{\mathrm{TM}} \mathrm{FA}$ (galactomannan) and a chosen fraction of Actigum $^{\mathrm{TM}}$ CS 6 (scleroglucan). To approach the diversity of real mucus, seven different gels with scleroglucan concentration from 0.5 to $2 \mathrm{wt} \%$ (by increments of $0.25 \%$ ) were prepared. The mixture was kept under agitation for $48 \mathrm{~h}$ at room temperature. After this period, a mass corresponding to $0.2 \mathrm{~mL}$ per $10 \mathrm{~mL}$ of di-sodium tetraborate at $0.02 \mathrm{M}$ (buffer component) was added to cross-link the galactomannan chains. The high molecular weight branched macromolecules of sugar, once cross-linked, build a gel matrix that mimicks the mucin network patterning the native mucus. The concentration of tetraborate (around $4 \times 10^{-4} \mathrm{~mol} / \mathrm{L}$ ) remains in the range $\left[2.5 \times 10^{-4}-7 \times 10^{-4}\right] \mathrm{mol} / \mathrm{L}$ found in the literature. ${ }^{1,27,33}$ It is notewothy that higher concentrations, as proposed by Ref. $27\left(4 \times 10^{-3} \mathrm{~mol} / \mathrm{L}\right)$ or more, lead to a gel with a jelly aspect. The agitation is kept for a few hours before storing the final mixture at $4^{\circ} \mathrm{C}$. Before performing the measurements, the gel is allowed to recover at room temperature and is fractionated into several $30 \mathrm{~mL}$ plastic vials.

Figure 2 presents the microstructure of the simulant using both an optical microscope and a scanning electron microscope for a solution of 2 wt $\%$ in Actigum $^{\mathrm{TM}}$. For the optical microscopy, droplets of mucus simulant were placed between two glass plates and loaded in an optical microscope (Leica DMRX, Germany) equipped with a $10 \times$ lens and different objectives (Fluotar, Germany) from $5 \times$ to $63 \times$. For scanning electron microscopy, small amounts of simulant were sampled and dehydrated by series of alcohol washings before being critical-point-dried. The dried samples were then metalized with a gold/palladium alloy before being observed with a scanning electron microscope (Hitachi S-4700) operating at $2.0 \mathrm{kV}$.

The microstructure is very similar to the one of bronchial mucus with a complex tangled network of worm-like filaments. ${ }^{34-36}$ From Figure 2, the filaments appear as linear chains at a larger scale (top) but at a smaller scale (bottom), they tangle to form a low viscosity network as reported by Voynow and Rubin ${ }^{37}$ for bronchial mucus. At the macroscale, the length of the filaments remains here of the order of $200 \mu \mathrm{m}$, while their thickness is rather constant and equal to $2.4 \mu \mathrm{m}$. At the microscale, the medium pore size is hardly accurately measurable with accuracy but it remains in accordance with the literature on human bronchial mucus: between ten and hundreds of $\mathrm{nm}$. For example, Kesimer et al. ${ }^{38}$ reported for real bronchial mucus that the typical length of mucins ranges between 0.2 and $1.5 \mu \mathrm{m}$ 


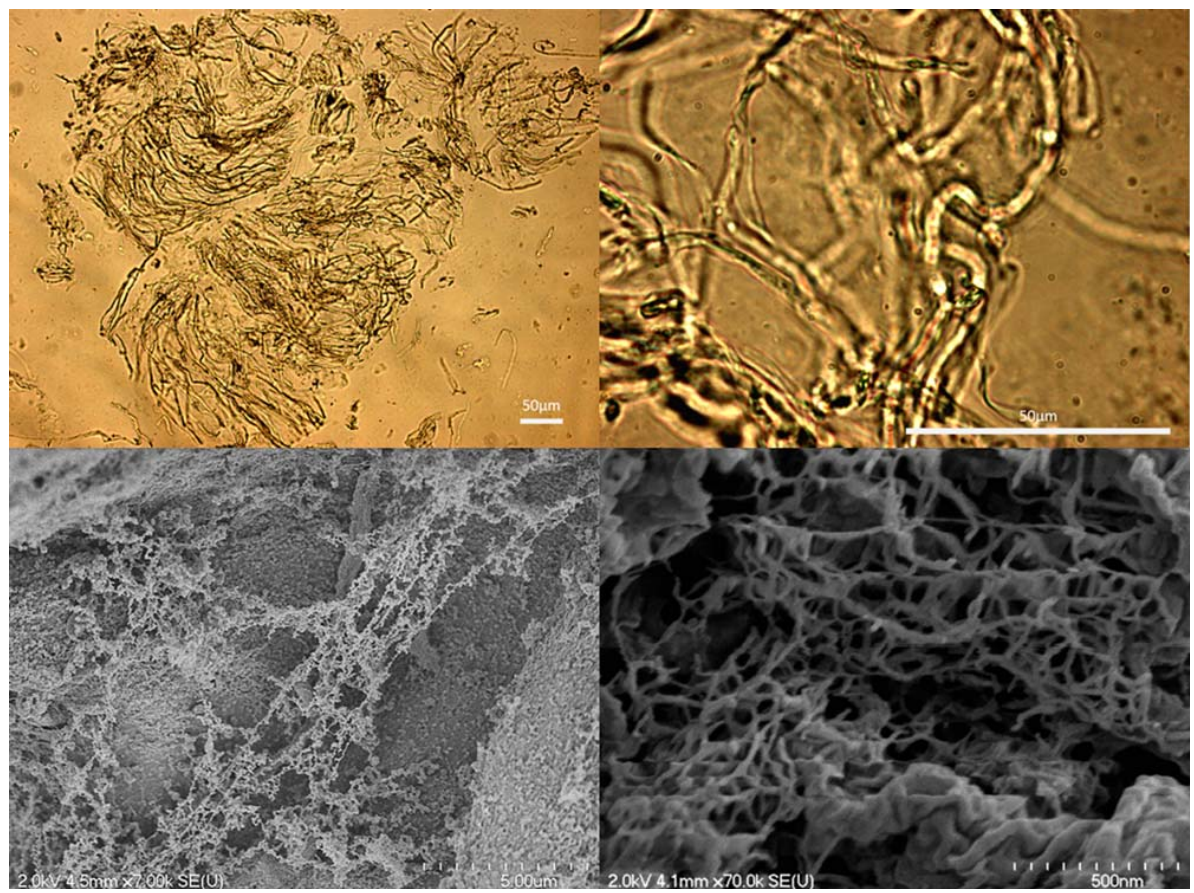

FIGURE 2. Structure of synthetic mucus ( $2 \mathrm{wt} \%$ of Actigum $^{\mathrm{TM}}$ ) using: an optical microscope (top, $10 \times$ and $63 \times$ ) and an scanning electron microscope (bottom).

depending on the type of mucins, while their typical width varies from 7 to $12 \mathrm{~nm}$. Nevertheless, in some respiratory diseases, such as severe asthma, there is an intermolecular cross-linking between mucin chains making the structure more complex and the length of some mucin chains much longer. ${ }^{37}$ A secondary polymer structure consisting of DNA and filamentous actin copolymers may coexist with the mucin chains for CF patients. This structure consists of thicker and longer polymer bundles dissociated from the mucin network and induces a decrease in elasticity compared to pure mucins. As shown in, ${ }^{37}$ the characteristic length of such a structure is close to the one observed in Figure 2 (top). More surprisingly, such longer bundles of fiber-like mucus strands have been observed for the stomach mucus from rabbits. ${ }^{39}$ It will be shown in Section " Results and Discussion", that such simulant mimic the main properties of bronchial mucus for CF patients. Increasing the concentration in Actigum ${ }^{\mathrm{TM}}$ (not shown here) leads to higher density regions without modifying the microstructure. One can attribute this to an unavoidable shrinkage happening during the drying process, as also reported in the literature. ${ }^{38}$

\section{MEASUREMENT TECHNIQUES}

\section{Rheological measurements}

The experiments were performed on a stress-controlled rheometer AR 550 (TA Instruments) equipped with a $50 \mathrm{~mm} /$ $2^{\circ}$ steel cone. Temperature was regulated by a Peltier plate. Dehydration was prevented by using a wet steel cover insuring a water saturated atmosphere around the sample. All the tests were realized at $20^{\circ} \mathrm{C}$ and $37^{\circ} \mathrm{C}$. No significant differences were noted depending on the temperature. Thus, series of isothermal experiments were performed at $20^{\circ} \mathrm{C}$ to limit dehydration issues.

The viscoelastic properties of the simulant unbroken structure were investigated through a series of dynamical shear experiments (Small Amplitude Oscillatory Shear). The results were interpreted from the evolution of the elastic and viscous moduli and the loss angle $\left(G^{\prime}, G^{\prime \prime}, \delta\right)$ in response to the sinusoidal load input. The stress dependency of the structure was observed via stress amplitude sweeps at a constant frequency (1 rad.s ${ }^{-1}$ ). More detailed rheological measurements will be presented in a further article.

\section{Densimetry measurements}

Density measurements have been performed using the DMA $5000 \mathrm{M}$ manufactured byAnton Paar GmbH. A $1 \mathrm{~mL}$ sample is placed in an U-shaped tube made in borosilicate glass, which is electronically oscillated at its characteristic frequency. This frequency varies with the density of the sample, which is deduced from the accurate measurement of the new characteristic frequency (U-shaped tube + sample) from a simple mathematical formula. The oscillations are measured by optical sensors. Temperature is controlled by a built-in Peltier thermostat. Two platine Pt 100 probes are used to measure the temperature of the sample, with an accuracy of $0.01^{\circ} \mathrm{C}$. For high viscosity samples, corrections are applied to avoid errors due to viscosity. It leads to highly repeatable and accurate density measurements: the standard deviations on the repeatability and accuracy of the density measurements are $10^{-6}$ and $5 \times 10^{-6} \mathrm{~g} / \mathrm{cm}^{3}$ respectively. 
The experimental protocol has been validated using distilled water with a standard deviation of $5 \times 10^{-6} \mathrm{~g} / \mathrm{cm}^{3}$ compared to the expected values between $19^{\circ} \mathrm{C}$ and $41^{\circ} \mathrm{C}$.

\section{Surface tension measurements}

The surface tension of the mucus simulant was measured by means of the du Noüy ring method using a semiautomatic tensiometer (Surface Tensiomat 21, Fisher Scientific). A platinum-iridium du Noüy ring (circumference $5.93 \mathrm{~cm}$, radius ratio between the ring and the wire $R /$ $r=53.2$ ) suspended to a counter-balanced lever arm was immersed in the mucus simulant samples before being driven upward by a torsion wire (radius $0.007^{\prime \prime}$ ) until the fluid film carried by the ring broke down. The apparent surface tension is determined from the force necessary to induce the breakage. Each measurement was repeated at least three times at room temperature $\left(23^{\circ} \mathrm{C}\right)$. This method was found to be more accurate than the Wilhelmy plate balance method described by Hamed and Fiegel. ${ }^{25}$

To validate the experimental protocol, preliminary tests have been performed using distilled water, acetone and toluene as calibration samples. For all these samples, the relative standard deviations and the repeatability remain better than $0.5 \%$.

\section{Thermal diffusivity and conductivity measurements}

Both thermal conductivity and diffusivity were measured using the THW-L1 Liquid Thermal Conductivity System from Thermtest Thermophysical Instruments. It measures simultaneously the thermal conductivity, $k$, and the thermal diffusivity, $\alpha$, based on the Transient Hot Wire (THW) method. Coupled with a system controlling the temperature (heat exchanger + thermostat bath circulator), this device allows a complete characterization of the thermal conductivity and diffusivity within the range $-40^{\circ} \mathrm{C}$ to $200^{\circ} \mathrm{C}$. The main advantage of this method for its application to complex fluids is its capacity to experimentally eliminate the error due to natural convection. The principle of the hot-wire method is based on an ideal and constant heat generation source, an infinitely long and thin continuous line, dissipating the heat into an infinite test medium. A constant current is supplied to a platinum wire (diameter $0.1 \mathrm{~mm}$, length $35 \mathrm{~mm}$ ) to generate the temperature rise. The wire serves as both the heat source and the temperature sensor. Heating the wire by Joule effect causes the variation of its resistance, thus its temperature is measured as a function of time using a Wheatstone bridge and a data acquisition system. A PT100 platinum resistance thermometer enables to measure independently the temperature of the sample. The THW sensor including the sample cell is made of stainless steel. The required sample volume is $50 \mathrm{~mL}$. The thermal conductivity value is determined from the heating power and the slope of temperature change in a logarithmic time scale. The thermal diffusivity is then evaluated through the thermal conductivity, temperature and heating power at a given time.

To validate the experimental protocol, preliminary tests have been performed using distilled water as the working

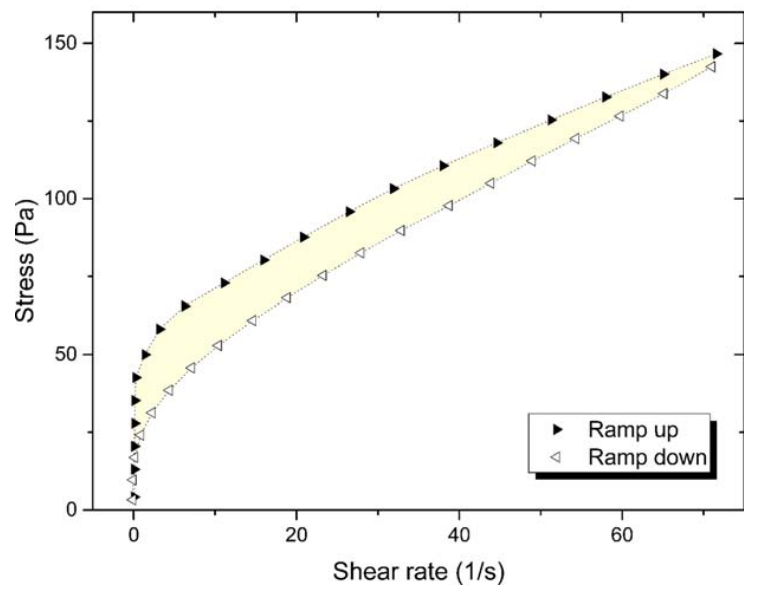

FIGURE 3. Example of rheogram for a 2 wt \% concentration in Actigum ${ }^{\mathrm{TM}}$ obtained at $20^{\circ} \mathrm{C}$. Continuous shear stress ramp (up and down) highlighting a hysteresis loop.

fluid between $20^{\circ} \mathrm{C}$ and $50^{\circ} \mathrm{C}$. For both thermal conductivity and diffusivity, the repeatability remains better than $1 \%$. The relative standard deviations for $k$ ranges between $0.1 \%$ at $20^{\circ} \mathrm{C}$ and $0.17 \%$ at $40.5^{\circ} \mathrm{C}$, while for $\alpha$, its remains at $0.1 \%$ for this range of temperatures.

\section{RESULTS AND DISCUSSION}

\section{Rheological properties}

Bronchial mucus is a complex biologic material with viscoelastic properties and a non-linear and time-dependent rheological behavior. ${ }^{7}$ These rheological properties have been widely observed qualitatively on real native, pathological or simulant mucus. ${ }^{13-23}$ In the present Section, the objective is to demonstrate that the present mucus formulation exhibits similar properties.

Figure 3 displays a typical rheogram obtained from continuous shear stress ramps (shear stress ramps of \pm 12.5 Pa.s ${ }^{-1}$ ) for a 2 wt $\%$ concentration in Actigum ${ }^{\mathrm{TM}}$ at $20^{\circ} \mathrm{C}$. A continuous shear stress up and down ramp test has been performed using different geometries (Couette, plate and cone) leading to similar results. A progressive departure from an elastic behavior to a shear-thinning behavior is observed beyond a stress threshold. This observation is the manifestation of the progressive yielding of the gel structure. The experimental data (up curve or down curve) are well fitted by a Herschel-Bulkley model with a flow index lower than 1. Nevertheless, one has to keep in mind that the result of this experiment is subjected to the timedependent behavior of the material. The yield stress observed here is not an inherent property of the material since the structural strength of the gel is time-dependent. As a consequence, in such a non steady flow measurement, the up curve apparent yield stress is above the down curve apparent yield stress. The characterization of such a non steady-state flow curve constitutes a first step toward a more complete characterization accounting for the steady state flow curve and the time-dependent behavior as well. The thixotropic property of the mucus simulant is simply 


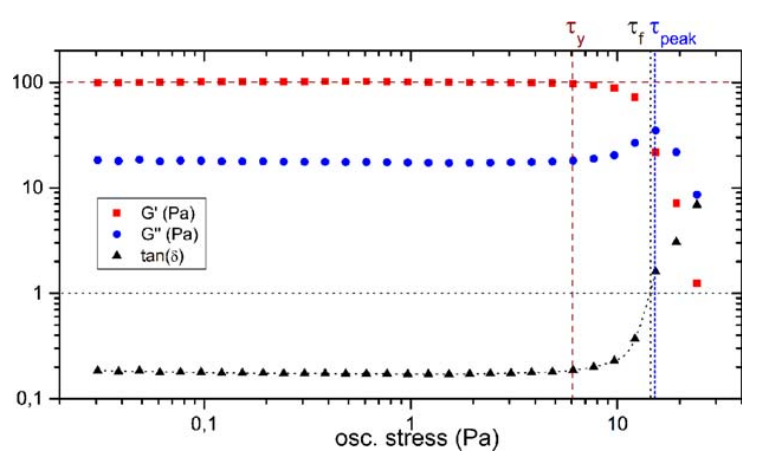

FIGURE 4. Small Amplitude Oscillatory Shear (SAOS) sweep test for a $1.5 \mathrm{wt} \%$ concentration in Actigum ${ }^{\mathrm{TM}}$ obtained at $20^{\circ} \mathrm{C}$ and a constant frequency of 1 rad.s ${ }^{-1}$. Elastic $G^{\prime}$ and viscous $G^{\prime \prime}$ moduli $(\mathrm{Pa})$ and the loss factor $(\tan \delta)$ as a function of the stress amplitude (Pa).

highlighted, on a purely qualitative manner, by the hysteresis loop in Figure 3 (yellow area).

Small Amplitude Oscillatory Shear (SAOS) sweep tests for a $2 \mathrm{wt} \%$ concentration in Actigum ${ }^{\mathrm{TM}}$ obtained at $20^{\circ} \mathrm{C}$ and a constant frequency of 1 rad.s $^{-1}$ have also been performed. Figure 4 presents the variations of the elastic $G^{\prime}$ and viscous $G^{\prime \prime}$ moduli and the loss factor $(\tan \delta$ ) as a function of the oscillating stress amplitude. A yield point, denoted $\tau_{\mathrm{y}}$, that delimits the linear viscoelastic domain can be deduced from a $5 \%$ departure from the plateau behavior of $G^{\prime}$. This is the stress limit above which the measurements no longer reflect the native structure since a structural breakdown starts to occur. Beyond the yield stress, the moduli are no longer constant due to the breakdown of the gel network. Just above this critical point, the stress is large enough to initiate the structure breakdown but too small to allow flowing. A flow point $\left(\tau_{\mathrm{f}}\right)$ is then identified as the shear stress corresponding to the moduli crossover, that is, the point above which the material becomes more viscous than elastic due to a critical structure breakdown.

Above the yield stress, the viscous modulus shows an overshoot before decreasing. According to Mezger ${ }^{40}$ this is a common phenomenon in gels. The increase of deformation energy inducing the overshoot could be related to a progressive collapse of the network due to structure components with some freedom of motion. The resulting friction would thus be responsible for the high dissipated energy (increase in $G^{\prime \prime}$ ) until the final breakdown occurs when $G^{\prime \prime}$

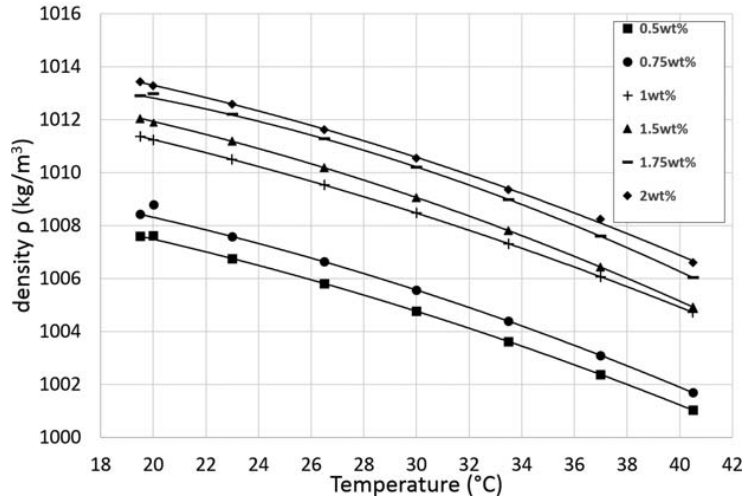

FIGURE 5. Influence of temperature and concentration in Actigum ${ }^{\mathrm{TM}}$ on the density $\rho$ of synthetic mucus.

displays a peak $\left(\tau_{\text {peak }}\right)$. This kind of behavior has been classified as a Type III ("weak strain overshoot") behavior by Hyun et al. ${ }^{41}$ A review of Hyun et al. ${ }^{42}$ emphasizes the very diverse possible structural causes of this behavior depending on the class of the soft material and claims the necessity to perform LAOS (Large Amplitude Oscillatory Shear sweep test) study to probe the meaning of this behavior.

Identical stress sweep tests have been performed on mucus simulants for the whole range of concentrations in Actigum $^{\mathrm{TM}}$. Similar curves are obtained for all concentrations (data not shown here) and the characteristic values are summarized in Table I. Viscous and elastic moduli and yield stresses all increase according to the polymer concentration. The overshoot is also observed at higher stress amplitudes for more concentrated samples. One could interpret this by the fact that more concentrated samples have a stronger network, which requires more energy to collapse. When the collapse begins, friction occurs and energy dissipation expresses itself by the $G^{\prime \prime}$ overshoot. The peak height is also amplified by the Actigum $^{\mathrm{TM}}$ concentration. When the structure starts collapsing, friction occurs between structural components. A more concentrated sample is expected to dissipate more friction energy as a result of its higher network density entailing more chain promiscuity and entanglements.

The plateau values of $G^{\prime}$ and $G^{\prime \prime}$ shown in Figure 4 or in Table I agree particularly well with the experimental values for human cystic fibrosis sputum displayed on Figure $5 \mathrm{a}$ in the review of Lai et al. ${ }^{7}$

TABLE I. Main Rheological Characteristics as a Function of wt $\%$ in Actigum ${ }^{\mathrm{TM}}$ Measured at $20^{\circ} \mathrm{C}$ for of 1 rad.s $^{-1}$. LVE Refers to the Linear Viscoelasticity Region

\begin{tabular}{|c|c|c|c|c|c|c|c|}
\hline $\begin{array}{l}\text { wt\% in } \\
\text { Actigum }^{T M}\end{array}$ & $G_{\text {LVE }}^{\prime}(\mathrm{Pa})$ & $G_{L V E}^{\prime}-5 \%(P a)$ & $G_{\text {LVE }}^{\prime \prime}(\mathrm{Pa})$ & $G_{\text {peak }}^{\prime \prime}(\mathrm{Pa})$ & $\tau_{y}(5 \%)(P a)$ & $\tau_{\mathrm{f}}(\mathrm{Pa})$ & $\tau_{\text {peak }}(\mathrm{Pa})$ \\
\hline 0.5 & 5.76 & 5.48 & 1.77 & 2.07 & 0.24 & 2.30 & 1.93 \\
\hline 0.75 & 14.36 & 13.64 & 3.68 & 5.86 & 2.43 & 5.20 & 4.84 \\
\hline 1 & 32.47 & 30.85 & 6.76 & 11.49 & 1.53 & 5.51 & 6.10 \\
\hline 1.25 & 39.57 & 37.59 & 12.90 & 16.01 & 2.43 & 8.90 & 9.66 \\
\hline 1.5 & 100.86 & 95.82 & 15.70 & 35.03 & 6.10 & 15.30 & 15.32 \\
\hline 1.75 & 108.35 & 102.93 & 18.86 & 43.34 & 7.68 & 18.72 & 12.17 \\
\hline 2 & 133.08 & 126.43 & 20.22 & 52.38 & 1.93 & 18.10 & 19.29 \\
\hline
\end{tabular}


TABLE II. Surface Tension as a Function of wt $\%$ in Actigum ${ }^{\mathrm{TM}}$ Measured at $23^{\circ} \mathrm{C}$

\begin{tabular}{|c|c|c|c|c|c|c|c|}
\hline wt\% in Actigum ${ }^{\mathrm{TM}}$ & 0.5 & 0.75 & 1 & 1.25 & 1.5 & 1.75 & 2 \\
\hline $\begin{array}{l}\text { Present measurements } \\
\left(10^{-3} \mathrm{~N} \cdot \mathrm{m}^{-1}\right)\end{array}$ & 71.6 & 72 & 75.4 & 78.4 & 86.1 & 86.6 & 89.9 \\
\hline Cystic fibrosis ${ }^{43,44}$ & & & & $\begin{array}{l}81.1-92.4 \\
79.1\end{array}$ & & & \\
\hline Chronic bronchitis $^{43}$ & & & & $72.1-84.8$ & & & \\
\hline Primary Ciliary Dyskenesia ${ }^{44}$ & & & & 82.9 & & & \\
\hline Bronchestasis $^{45}$ & & & & 86 & & & \\
\hline Respiratory Tract Infection ${ }^{46}$ & & & & 86.47 & & & \\
\hline Synthetic mucus ${ }^{25,47}$ & & & & $\begin{array}{l}53.3-95 \\
\sim 72\end{array}$ & & & \\
\hline
\end{tabular}

\section{Surface tension}

Surface tension of synthetic mucus with concentration in Actigum $^{\mathrm{TM}}$ varying between 0.5 and 2 wt \% has been measured by a du Nouy ring tensiometer at $23^{\circ} \mathrm{C}$. The results are compared to former results obtained on real mucus ${ }^{43-46}$ or mucus simulants ${ }^{25,47}$ in Table II. The surface tension is particularly interesting since it is closely related to the wettability and adhesiveness of bronchial mucus on the epithelium surface. It directly influences the efficiency of ciliary and cough clearance on the mucus transport.

At low concentrations in Actigum ${ }^{\mathrm{TM}}$, the present values are close to that of pure water, whose surface tension slightly decreases from $72.8 \mathrm{mN} \cdot \mathrm{m}^{-1}$ at $20^{\circ} \mathrm{C}$ to $70 \mathrm{mN} \cdot \mathrm{m}^{-1}$ at $37^{\circ} \mathrm{C}$ As already reported by Hamed and Fiegel, ${ }^{25}$ surface tension increases with cross-linking due here to an increase of the concentration in Actigum $^{\mathrm{TM}}$. Albers et al. ${ }^{43}$ also observed higher surface tensions for more solid-like sputum surfaces. The values reported here are significantly different from those obtained for native mucus, which contains surface-active molecules. This kind of surfactant considerably lowers the surface tension of mucus down to $30-34 \mathrm{mN} . \mathrm{m}^{-1}$ approximately. ${ }^{48}$

For high concentrations, the results have to be considered with special care as the du Nouy ring method requires some corrections to account for the complex shape of the meniscus during the ring detachment. As discussed in, ${ }^{25}$ such a correction exists only for Newtonian fluids and cannot be applied here leading to less reliable values of surface tension.

\section{Other properties}

To the best of the author's knowledge, the density, heat capacity and thermal diffusivity (or conductivity) of mucus simulants or sputum have not been considered in the literature. As it will be shown below, these properties take different values compared to those of pure water. They have to be measured carefully for the different concentrations $\varphi$ in Actigum $^{\mathrm{TM}}$ at the body temperature. From a thermal point, the respiratory system also plays the role of humidifier and heater for the inspired gas. Thomachot et al. ${ }^{49}$ measured the minimal and maximal temperatures in the trachea of 10 healthy patients. For an ambient air temperature around $23^{\circ} \mathrm{C}$ and mean body temperatures equal to $37.8^{\circ} \mathrm{C}$, temperatures in the upper part of the trachea vary between 30 and $33^{\circ} \mathrm{C}$. Considering the whole upper airways (nose, mouth, larynx...), they can vary between the ambient temperature and $33^{\circ} \mathrm{C}$. It appears then also important to measure the thermal properties of mucus simulants for a temperature range between $20^{\circ} \mathrm{C}$ and $40^{\circ} \mathrm{C}$ (patients with fever). It will provide a very useful database for future advanced numerical modelings dedicated to the transport of bronchial mucus in the respiratory system by mucociliary clearance or helping clearance devices.

Figure 5 presents the evolution of the density $\rho$ of mucus simulants as a function of temperature. As for pure water, $\rho$ decreases quadratically with temperature. For example, for $\varphi=2$ wt $\%$, one gets:
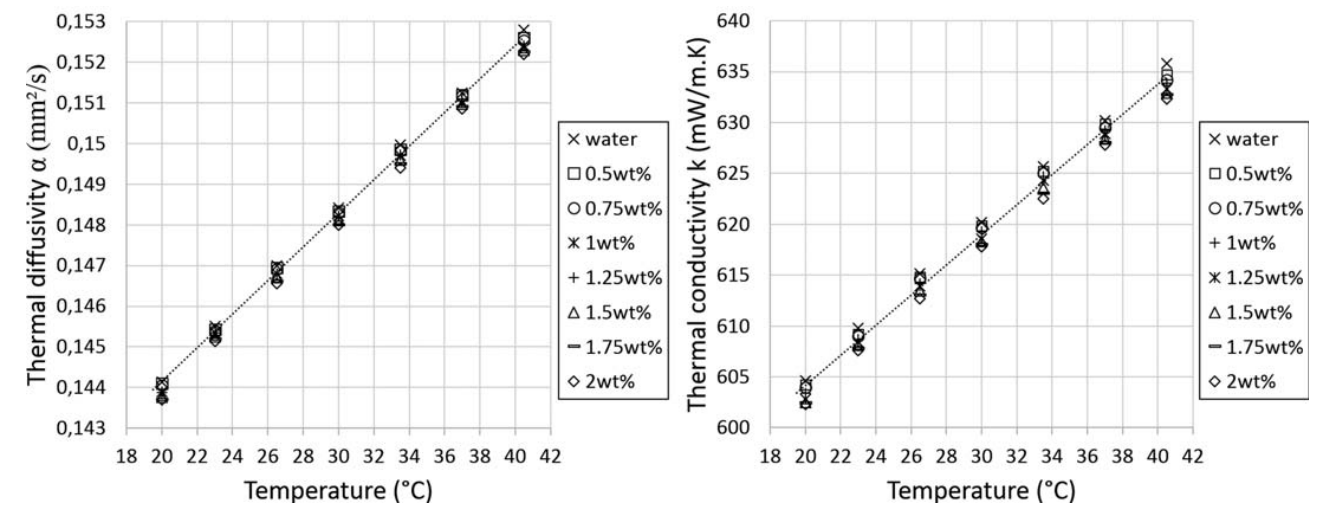

FIGURE 6. Influence of temperature and concentration in Actigum ${ }^{\mathrm{TM}}$ on the (a) thermal diffusivity $\alpha$ and (b) thermal conductivity $k$ of synthetic mucus. 


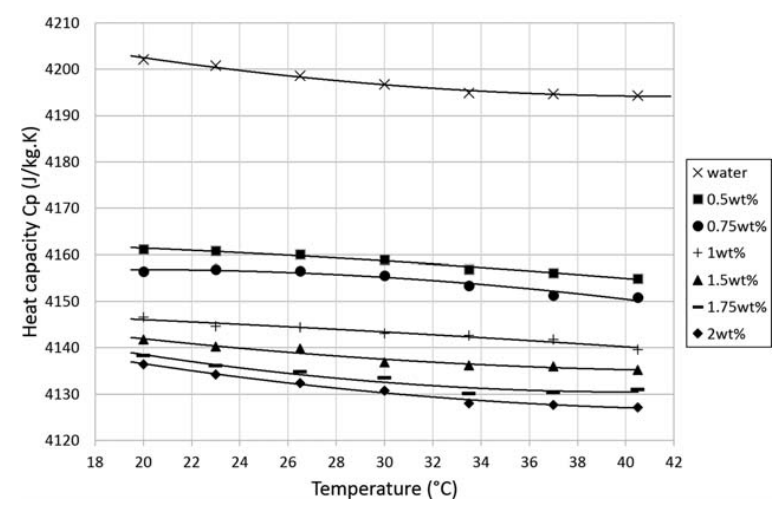

FIGURE 7. Influence of temperature and concentration in Actigum $^{\mathrm{TM}}$ on the heat capacity $C p$ of synthetic mucus.

$$
\rho(T, \varphi=2 \%)=-0.0049 T^{2}-0.0277 T+1015.8
$$

with $\mathrm{T}$ in ${ }^{\circ} \mathrm{C}$. Eq. (1) is to be compared to the following expression for pure water:

$$
\rho(T, \text { water })=-0.0041 T^{2}-0.0574 T+1001.1
$$

As expected also, $\rho$ is also an increasing function of the concentration $\varphi$ in Actigum $^{\mathrm{TM}}$. For the lower concentration, $\varphi$ $=0.5 \mathrm{wt} \%, \rho$ is increased by around $0.9 \%$ compared to pure water at the same temperature. All results are fitted by the following quadratic relationship giving $\rho$ as a function of the concentration $\varphi$ in Actigum ${ }^{\mathrm{TM}}$ for a given prescribed temperature $T\left({ }^{\circ} \mathrm{C}\right)$ :

$$
\left\{\begin{array}{c}
\rho(\forall T, \varphi)=a \varphi^{2}+b \varphi+c(\varphi) \\
c(\varphi)=-1.41 \varphi^{2}+7.495 \varphi+1006.6 \\
a=-0.0045 \text { and } b=-0.045\left(R^{2}=0.9845\right)
\end{array}\right.
$$

Figure 6 presents the variations of thermal diffusivity and conductivity versus the temperature. Both quantities increases linearly with temperature as in the case of pure water. The influence of the concentration $\varphi$ in Actigum $^{\mathrm{TM}}$ is almost negligible. The thermal conductivity (resp. diffusivity) of pure water is $0.4 \%$ (resp. $0.3 \%$ ) higher (resp. lower) in average than that for $\varphi=2$ wt $\%$.

The heat capacity at constant pressure has been deduced from the previous measurements through the relation: $C_{p}=k /(\alpha \rho)$. Figure 7 displays the distribution of the heat capacity versus temperature for different values of $\varphi$. Whatever the concentration in Actigum ${ }^{\mathrm{TM}}$, the heat capacity decreases quadratically with temperature. As expected, taking into account the fact that the heat capacity of Actigum ${ }^{\mathrm{TM}}$ is lower than that of pure water, the heat capacity decreases also with $\varphi$. At a given imposed temperature, $C_{p}$ decreases by $1.6 \%$ between pure water and the $\varphi=2$ wt $\%$ simulant.

Note that the effect of aging on the heat capacity of mucus simulant has also been considered. There is no noticeable difference between measurements performed with "fresh" simulant or with simulant prepared 4 days before and stored in a fridge at $4^{\circ} \mathrm{C}$.

\section{CONCLUSION}

Bronchial mucus plays a crucial role in the protection of the respiratory system and can be seen as a key-element for better understanding and treatment of chronic respiratory diseases such as severe asthma or cystic fibrosis. The airway clearance function is a complex phenomenon directly linked to the thermophysical properties of the mucus layer coating the airways. The difficulty to collect and exhaustively test samples from a same mucus collection first motivated the choice to work here on mucus simulants. The composition and experimental protocol to prepare this synthetic mucus were proposed by. ${ }^{33}$ This gel is mainly composed of water, $\mathrm{NaCl}$, Viscogum ${ }^{\mathrm{TM}} \mathrm{FA}$ (galactomannan) and a variable fraction of Actigum $^{\mathrm{TM}}$ CS 6 (scleroglucan) to mimic a large range of mucus consistencies.

The rheological properties at rest were investigated using small amplitude oscillatory shear tests. They revealed that mucus simulant behaves as a gel within a defined linear viscoelastic region and as a viscoelastic liquid above the yield stress zone. To characterize the behavior of mucus in response to in vivo shearing, continuous ramp flow tests have also been performed. The flow curves revealed the yield stress and shear-thinning behavior of the mucus simulant as well as a hysteresis loop between the up and down curves that qualitatively accounts for the time-dependent (thixotropic) behavior.

Measurements of the surface tension of mucus simulants also measured here, were in good agreement with previous published data. The present synthetic mucus described as the most appropriate one in the literature, is here shown to mimic precisely the surface tension of real mucus for different pathologies ranging from cystic fibrosis to respiratory tract infection. Future measurements of the contact angle should enable to determine experimentally the adhesiveness of such mucus simulants.

Thermal properties, namely thermal diffusivity, thermal conductivity and heat capacity, are quite comparable to those of pure water in the range of tested temperature (20$40^{\circ} \mathrm{C}$ ). Thermal diffusivity and thermal conductivity increases linearly by respectively $6 \%$ and $5 \%$, while the heat capacity decreases by only $0.3 \%$ between $20^{\circ} \mathrm{C}$ and $40^{\circ} \mathrm{C}$.

\section{ACKNOWLEDGMENTS}

O. Lafforgue would like to thank the company Physio-Assist and the Association Nationale de la Recherche et de la Technologie for the CIFRE grant 2014-1287. S. Poncet gratefully acknowledges the support of the Natural Sciences and Engineering Research Council of Canada through the Discovery Grant (RGPIN-2015-06512) and Canadian Foundation for Innovation (Grant 34582) for the provision of most measurement facilities through the John R. Evans Leaders fund.

\section{REFERENCES}

1. Zahm JM, King M, Duvivier C, Pierrot D, Girod S, Puchelle E. Role of simulated repetitive coughing in mucus clearance. Eur Respir $J$ 1991;4:311-315.

2. Cone RA. Barrier properties of mucus. Adv Drug Deliv Rev 2009; 61:75-85. 
3. Flume PA, Robinson KA, O'Sullivan BP, Finder JD, Vender RL, Willey-Courand DB, White TB, Marshall BC. Cystic fibrosis pulmonary guidelines: Airway clearance therapies. Respir Care 2009;54: 522-537.

4. Majima Y. Mucoactive medications and airway disease. Paediatr Respir Rev 2002;3:104-149.

5. Cantin AM, Bacon M, Berthiaume Y. Mechanical airway clearance using the Frequencer electro-acoustical transducer in cystic fibrosis. Clin Invest Med 2006; 29: 159-165.

6. Benkoussas H, Seyssiecq-Guarente I, Poncet S. Étude expérimentale de l'influence de la dépression mécanique sur le transport et le comportement rhéologique du mucus bronchique synthétique dans une trachée artificielle. $2 e$ Conférence Internationale de Mécanique (ICM'15), Constantine, November 25-26, 2015.

7. Lai SK, Wang YY, Wirtz D, Hanes J. Micro- and macrorheology of mucus. Adv Drug Deliv Rev 2009;61:86-100.

8. Vasquez PA, Forest MG. Chapter 2: Complex fluids and soft structures in the human body. In: Spagnolie SE, editor. Complex Fluid in Biological Systems. New York: Springer Science; 2015. p 53110.

9. Thornton DJ, Sheehan JK. From mucins to mucus: Toward a more coherent understanding of this essential barrier. Proc Am Thorac Soc 2004;1:54-61.

10. King M. Mucus and its role in airway clearance and cytoprotection. In: Q. Hamid (ed.), Physiologic Basis of Respiratory Disease. Hamilton (Ontario, Canada): BC Decker Inc; 2005;409-416.

11. Boat $T$, Cheng $P$, Leigh $M$. Biochemistry of mucus. In: Lenfant $C$ editor. Lung Biology in Health and Disease. New York: Marce Dekker; 1994. p 217282.

12. Verdugo P. Supramolecular dynamics of mucus. Cold Spring Harb Perspect Med 2012;2:1-15.

13. Rubin BK. Mucus structure and properties in cystic fibrosis. Paediatr Respir Rev 2007;8:4-7.

14. Denton R. Rheology of human lung mucus. Ann N Y Acad Sci 1963;106:746-754.

15. Davis SS, Dippy JE. The rheological properties of sputum. Biorheology 1969;6:11-21.

16. Litt M. Mucus rheology. Relevance to mucociliary clearance. Arch Intern Med 1970;126:417-423.

17. Puchelle E, Zahm JM, Havez R. Biochemical and rheological data in sputum. Relationship between the biochemical constituents and the rheological properties of sputum. Bull PhysioPatho Respir 1973;9:237-257.

18. Puchelle E, Zahm JM, Duvivier C. Spinability of bronchial mucus. Relationship with viscoelasticity and mucous transport properties. Biorheology 1983;20:239-249.

19. Puchelle E, Zahm JM, Duvivier C, Didelon J, Jacquot J, Quemada D. Elastothixotropic properties of bronchial mucus and polyme analogs. I. Experimental results. Biorheology 1985;22:415-423.

20. Puchelle E, Zahm JM, Quemada D. Rheological properties controlling mucociliary frequency and respiratory mucus transport. Biorheology 1987;24:557-563.

21. Rubin BK, Ramirez O, Zayas JG, Finegan B, King M. Collection and analysis of respiratory mucus from subjects without lung disease. Am Rev Respir Dis 1990;141:1040-1043.

22. Nielsen H, Hvidt S, Sheils CA, Janmey PA. Elastic contributions dominate the viscoelastic properties of sputum from cystic fibrosis patients. Biophys Chem 2004;112:193-200.

23. Puchelle E, Jacquot J, Beck G, Zahm JM, Galabert C. Rheological and transport properties of airway secretions in cystic fibrosisrelationships with the degree of infection and severity of the disease. Eur J Clin Invest 1985;15:389-394.

24. Kollerstrom N, Lord PW, Whimster WF. A difference in the composition of bronchial mucus between smokers and non-smokers. Thorax 1977;32:155-159.

25. Hamed R, Fiegel J. Synthetic tracheal mucus with native rheological and surface tension properties. J Biomed Mater Res A 2014, 102:1788-1798.

26. King M, Gilboa A, Meyer FA, Silberberg A. On the transport of mucus and its rheologic simulants in ciliated systems. Am Rev Respir Dis 1974:110:740-745.

27. Anwarul Hasan MD, Lange CF, King ML. Effect of artificial mucus properties on the characteristics of airbone bioaerosol droplet generated during simulated coughing. J NonNewton Fluid Mechan 2010:165:1431-1441.

28. King M, Brock G, Lundell C. Clearance of mucus by simulated cough. J Appl Physiol 1985;58:1776-1782.

29. Hassan AA Evrensel AC Krumpe PE Clearance of viscoelastic mucus simulant with airflow in a rectangular channel, an experimental study. Technol Health Care 2006;14:1-11.

30. Madsen F, Eberth K, Smart J. A rheological evaluation of various mucus gels for use in in-vitro mucoadhesion testing. J Pharm Sci 1996;2:563-566.

31. Banerjee R, Bellare J, Puniyani R. Effect of phospholipid mixtures and surfactant formulations on rheology of polymeric gels, simulating mucus, at shear rates experienced in the tracheobronchial tree. Biochem Eng J 2001;7:195-200.

32. Shah $S$, Fung $K$, Brim $S$, Rubin BK. An in vitro evaluation of the effectiveness of endotracheal suction catheters. Chest 2005;128: 3699-3704.

33. Zahm JM. Tests effectués avec I'appareil SIMEOX. INSERM report, 2007.

34. Suk JS, Lai SK, Boylan NJ, Dawson MR, Hanes Boyle MP. Rapid transport of muco-inert nanoparticles in cystic fibrosis sputum treated with n-acetyl cysteine. Nanomedicine 2011;6:365-375.

35. Manzenreiter $R$, Kienberger $F$, Marcos V, Schilcher $K$ Krautgartner WD, Obermayer A, Huml M, Stoiber W, Hector A Griese M, Hannig M, Studnicka M, Vitkov L, Hartl D. Ultrastructural characterization of cystic fibrosis sputum using atomic force and scanning electron microscopy. J Cyst Fibros 2012;11:84-92.

36. Schuster BS, Jung Soo S, Woodworth GF, Hanes J. Nanoparticle diffusion in respiratory mucus from humans without lung disease. Biomaterials 2013;34:3439-3446.

37. Voynow JA, Rubin BK. Mucins, Mucus, and Sputum. Chest 2009 135:505-512.

38. Kesimer M, Ehre C, Burns KA, Davis CW, Sheehan JK, Pickles RJ. Molecular organization of the mucins and glycocalyx underlying mucus transport over mucosal surfaces of the airways. Mucosal Immunol 2013:6:379-392.

39. Bansil R, Celli JP, Hardcastle JM, Turner BS. The influence of mucus microstructure and rheology in Helicobacter pylori infection. Front Immunol 2013;4:1-12.

40. Mezger TG. The Rheology Handbook: For Users of Rotational and Oscillatory Rheometers. $4^{\text {th }}$ Edition, Vincentz Network GmbH \& Co. (Ed.), European Coatings Tech Files, Hanover (Germany), 2014.

41. Hyun K, Kim SH, Ahn KH, Lee SJ. Large amplitude oscillatory shear as a way to classify the complex fluids. J Nonnewton Fluid Mech 2002;107:51-65.

42. Hyun $\mathrm{K}$, Wilhelm $\mathrm{M}$, Klein $\mathrm{CO}$, Cho $\mathrm{KS}$, Nam JG, Ahn $\mathrm{KH}$, Lee $\mathrm{SJ}$ Ewoldt $\mathrm{RH}$, McKinley $\mathrm{GH}$. A review of nonlinear oscillatory shear tests: Analysis and application of large amplitude oscillatory shear (laos). Progr Polym Sci 2011;36:1697-1753.

43. Albers GM, Tomkiewicz RP, May MK, Ramirez OE, Rubin BK. Ring distraction technique for measuring surface tension of sputum: Relationship to sputum clearability. J Appl Physiol 1996;81:2690-2695.

44. Bush A, Payne D, Pike S, Jenkins C, Henke MO, Rubin BK. Mucus properties in children with primary ciliary dyskinesia: Comparison with cystic fibrosis. Chest 2006;129:118-123.

45. Daviskas $\mathrm{E}$, Anderson SD, Young $\mathrm{IH}$. Effect of mannitol and repetitive coughing on the sputum properties in bronchiectasis. Respir Med 2010;104:371-377.

46. Bennett WD, Kala A, Duckworth $\mathrm{H}$, Zeman KL, Wu J, Henderson A Yopp M, Rubin BK. Effect of a single $1200 \mathrm{mg}$ dose of Mucinex ${ }^{\circledR}$ on mucociliary and cough clearance during an acute respiratory tract infection. Respir Med 2015;109:1476-1483.

47. Schenck DM, Fiegel J. Tensiometric and phase domain behavio of lung surfactant on mucus-like viscoelastic hydrogels. Appl Mater Interfaces 2016;8:5917-5928.

48. Im Hof V, Gehr P, Gerber V, Lee M, Schurch S. In vivo determination of surface tension in the horse trachea and in vitro model studies. Respir Physiol 1997;109:81-93.

49. Thomachot L, Viviand X, Lagier P, Dejode JM, Albanèse J, Martin C. Measurement of tracheal temperature is not a reliable index of total respiratory heat loss in mechanically ventilated patients. Critical Care 2001;5:24-30 